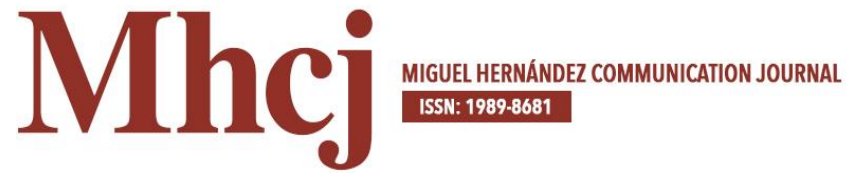

\title{
Trasladar la partida: Modelos y estrategias de la industria cinematográfica frente a la adaptación de un videojuego
}

\author{
Dr. Mario-Paul Martínez Fabre| mario.martinez@umh.es \\ Universidad Miguel Hernández
}

\author{
Palabras clave \\ Videojuegos, cine, adaptación, Hollywood, \\ intertextualidad \\ Sumario \\ 1. Introducción 2. Marco de estudio 3. Resultados \\ 4. Discusión 5. Conclusiones 6. Bibliografía y \\ filmografía 7. Notas
}

\begin{abstract}
Resumen
La 'adaptación' cinematográfica es un término que, hasta hace relativamente poco, el cine reservaba a la literatura, al teatro y a su propia "autorrevisión" en forma de remake. Hoy esta operación se ha impuesto con fuerza en el cine
\end{abstract} contemporáneo, especialmente en lo que respecta a la producción sustraída de los objetos de la cultura de masas: cómics, series de TV, atracciones de parques temáticos o, como este artículo analiza, videojuegos, se erigen también como una fuente importante de producción para las películas abanderadas por Hollywood. Resulta necesario examinar cómo estas adaptaciones de videojuegos han cobrado fuerza en el ámbito audiovisual (especialmente en el cine mainstream), cuál es su trayectoria, y como trasladan su narrativa y su lenguaje a la pantalla de cine. Una tarea que se llevará a cabo analizando a las principales precursoras, a las grandes apuestas de la industria del cine (alto presupuesto) y las adaptaciones de medio o bajo presupuesto (orientadas a un público más entendido), teniendo presente aspectos como las particularidades de la plataforma a adaptar, las diferentes condiciones del videojuego $\mathrm{u}$ otros aspectos como la intertextualidad, la intermedialidad o la autorrenferencia que, de un modo u otro, también se entrelazan con las particularidades del cine espectáculo contemporáneo y sus fórmulas de rentabilidad y seriación. La adaptación Lara Croft: Tomb Raider, nos servirá como caso de estudio para dar cuenta de cómo, todas estas estrategias, se aplican a una adaptación exitosa en cuanto a resultados de taquilla, narratividad y formulación estética.

\section{Cómo citar este texto:}

Mario-Paul Martínez Fabre (2017): “Trasladar la partida: Modelos y estrategias de la industria cinematográfica frente a la adaptación de un videojuego", en Miguel Hernández Communication Journal, nº, pp. 205 a 229. Universidad Miguel Hernández, UMH (ElcheAlicante). Recuperado el $\_$de de 20 de: Џink del artículo en 


\title{
Porting the game: Models and strategies of the film industry against the adaptation of a video game
}

\author{
Dr. Mario-Paul Martínez Fabre| mario.martinez@umh.es \\ Universidad Miguel Hernández
}

\author{
Keywords \\ Video games, cinema, adaptation, Hollywood, \\ intertextuality \\ Summary \\ 1. Introduction 2. Framework 3. Results \\ 4. Discussion 5. Conclusions 6. Bibliography and
}

\section{Abstract}

The film 'adaptation' is, until recently, a term that the film industry reserved to literature, theater and its own "selfrevision" as a remake. Today this operation has been imposed considerably in contemporary cinema, especially as regards to the subtracted production from objects of mass culture: comics, TV series, theme park rides or, as this article discusses, video games, also it stands as an important source of production for Hollywood movie bearers.

It is necessary to examine how these video game adaptations have gained strength in the audiovisual field (especially in mainstream cinema), what is their path, and how they move their narrative and language to the big screen. A task to be carried out by analyzing the main precursor to the biggest bets of the film industry (high budget) and adaptations of medium or low budget (aimed at a more understanding public), taking into account aspects such as the characteristics of the platform to adapt, the different conditions of the game or other aspects such as intertextuality, intermediality or the self-references that, one way or another, is also intertwined with the peculiarities of contemporary spectacle cinema and formulas profitability and serialization. The adaptation Lara Croft: Tomb Raider, will serve as a case study to explain how, all these strategies, are applied in a successful adaptation in terms of box office results, narrativity and aesthetic formulation.

\section{How to cite this text:}

Mario-Paul Martínez Fabre (2017): "Porting the game: Models and strategies of the film industry against the adaptation of a video game", en Miguel Hernández Communication Journal, n8, pp. 205 to 229. Universidad Miguel Hernández, UMH (Elche-Alicante). Accessed 20 _ in: [paper link in mhjournal.org] 


\section{Introducción}

El propósito de este artículo es concretar cuáles son los conceptos básicos que definen una adaptación cinematográfica y como éstos se conectan con el texto, el lenguaje y los recursos que ofrece un videojuego al trasladarlo a la pantalla de cine. Para ello, y tras una breve recapitulación de las distintas etapas y grupos formados por las adaptaciones cinematográficas de los juegos digitales, se desgranará mediante un caso práctico (Lara Croft: Tomb Raider) cuáles son las pautas que las hacen funcionar y cuáles las intenciones que guían a sus productoras en el mercado.

\section{Marco de estudio}

\subsection{Hipótesis}

De tal manera, la hipótesis de este texto pretende certificar a) la existencia de un fenómeno como es la adaptación cinematográfica de un videojuego, y b) demostrar como, para realizar tal adaptación, la industria del cine ha adquirido ciertos recursos y prácticas generales afines a los modelos de consumo de la cultura de masas.

\subsection{Delimitaciones}

Para examinar cuáles son las estrategias de formación, y crecimiento de un producto como la adaptación cinematográfica de un videojuego -sea frente al mercado y la industria, o frente a sus propios traslados creativos-, se empleará como marco de referencia la cartelera internacional. Es decir, aquellas adaptaciones de un videojuego que han sido estrenadas en, al menos, dos países de diferente región del globo. Esto no incluye, por tanto, ni lanzamientos directos para TV o DVD; ni films solamente estrenados en su propio mercado de origen. Tampoco derivados como las películas realizadas por fans que emplean nuevos recursos fílmicos y han cobrado fuerza a través de Internet como, por ejemplo, las películas machinima (películas realizadas usando los motores y las escenas de los videojuegos).

Las cuestiones de esta elección responden, principalmente, a la necesidad de abordar el estudio desde un marco general significativo en lo que respecta a la industria y al mercado, y a la incidencia de estas películas en lo social y en el ámbito la cultura de masas. Al mismo tiempo, a la necesidad de comprender que los estrenos directos de TV, video/DVD o los subproductos difundidos en 
Internet reflejan, a su propio nivel (financiero, creativo), los patrones establecidos por las adaptaciones internacionales estudiadas.

\subsection{Metodología}

La metodología de investigación empleada como base es el análisis de contenido; contando, además, con las aportaciones surgidas en el seno de los estudios de la lúdica digital (Estudios del Juego o Game Studies¹), y con las aportaciones del área de los Estudios de Cine. Asimismo, y en grado más plural o catalizador, con las contribuciones que pueden ampliar estos puntos de vista desde la perspectiva que ofrecen los Estudios Visuales. Es decir, desde la mirada que nos permite localizar las adaptaciones de videojuegos en el contexto más amplio de los procesos creadores de significado de su entorno cultural.

Los Estudios del Juego o Game Studies representan una disciplina académica que se ocupa fundamentalmente de analizar el contexto, diseño e interacción de un juego. Se trata de un campo de estudio heterogéneo y empíricamente variado, que se nutre de las ciencias sociales (fines pedagógicos, etnológicos, etc.), de las humanidades (estética, historia, etc.) y del enfoque de la industria y la ingeniería (la ludología en su avance tecnológico), para dar cuenta de cómo un juego, en el caso que nos ocupa un videojuego, además de tener un papel protagonista en el mercado del ocio, es un producto cultural con un significado implícito.

En el caso concreto de este artículo, los Estudios de Juego nos servirán primero, para localizar y explicar ciertos recursos específicos del videojuego (como pueden ser su jugabilidad, su dinámica, su tipo de narrativa etc.), y segundo para conectar el análisis de estos recursos, con los obtenidos en una adaptación cinematográfica de videojuego como es Lara Croft: Tomb Raider desde la perspectiva de los Estudios de Cine.

El empleo metodológico de los Estudios de Cine implica, en este artículo, una tarea con mayores matices. Esto es así porque, aunque podemos encontrar una bibliografía que trata el tema del cine y del videojuego, no existen publicaciones que presenten un método específico para el análisis de este tipo de películas. Por tanto, y asumiendo que el cine de videojuegos y el principal caso de estudio, Lara Croft: Tomb Raider, subsisten esencialmente bajo las reglas de una cultura de masas y de una industria de tradición norteamericana, se decidió emplear textos de cabecera que, por un lado, hiciesen referencia a este tipo de crítica sobre el cine (como, por ejemplo y entre muchos otros, Cómo Analizar un Film de Casetti y de Chio, El Arte Cinematográfico, de Bordwell y Thompson), 
y que, por otro, también tuviesen en cuenta la naturaleza 'impura' e intertextual del cine (como, por ejemplo, el libro Teorías del Cine de Stam).

En tercer lugar y como una metodología catalizadora, se acudió a la rama de los Estudios Visuales. Esta corriente metodológica, tal y como se apuntaba, permite localizar al cine de videojuegos en un contexto más amplio de significados sin dejar de lado las experiencias visuales "no-mediadas" que nos puede aportar lo cotidiano o lo popular.

De tal manera, el uso en este artículo de los Estudios Visuales se debe también a su capacidad para desplegar una metodología "interdisciplinar" capaz de analizar los casos de estudio y la adaptación Lara Croft: Tomb Raider, no solo desde su carácter intertextual e intermedial (muy marcado por la potenciación tecnológica aludida por autores como José Luis Brea o Moxey), sino también desde una perspectiva donde lo visual, más allá de su estudio formal (Estudios de Imagen, Estudios de Media, por ejemplo), se debe de igual manera al estudio de una construcción social y a los valores de quienes crean, manipulan y consumen estas imágenes.

Finalmente, no puede obviarse que la relación directa que se establece con los casos de estudio generó, en el consumo de los mismos, una metodología práctica especialmente versada en la experiencia del jugar y del ver, apoyada también en una serie de fuentes bibliográficas y documentales sobre el cine y los videojuegos (bases de datos como <www.IMDB.com>, $<$ www.boxofficemojo.com $>, \quad<$ www.filmaffinity.com $>$ $<\underline{\text { www.rottentomatoes.com }}>,<$ www.gamasutra.com $>$ ).

\section{La adaptación cinematográfica de un videojuego}

Como producto cinematográfico, la adaptación equivale a la interpretación o traducción de un texto ya existente, en una nueva versión destinada a las salas de cine. Se trata de un proceso expuesto a las controversias de la 'fidelidad' -y no siempre con resultados convincentes- dada la inevitable mutación de forma y fondo a la que se somete el texto original al ser adaptado: sea al traducir los elementos pertenecientes a la historia (adiciones o supresiones narrativas, condensaciones y re-disposiciones de los acontecimientos, etc.), o al traducir los elementos modales o enunciativos propios de cada lenguaje (variaciones del punto de vista, sobre los elementos descriptivos, etc.) (McFarlane, 1996).

La adaptación cinematográfica de un videojuego no dista de los procesos de interpretación básicos que realiza el cine para adaptar otras artes como pueden 
ser el teatro o la novela, puesto que éstas y el mismo film son también artes de acción. Todas ellas poseen la capacidad de organizar acontecimientos, acciones, sobre una estructura de base. Parafraseando a Eco (1970: 194-200), todo texto narrativo -sea cual sea su naturaleza- expresa, a través de un lenguaje propio, un contenido que es básico y puede servir a múltiples sistemas significativos. Desde esta perspectiva, la figura del adaptador cinematográfico encuentra en las pantallas de un videojuego lo que encuentra en las páginas de un texto teatral o las viñetas de un cómic: un material inspirador sobre el que construir algo nuevo. Sus principales directrices serán el manejo de las divergencias formales entre ambas partes (original y adaptación) y la necesidad de efectuar los sacrificios narrativos o estéticos necesarios para la transposición de ambas ficciones.

Este catálogo de soluciones, además, se verá afectado especialmente por los espacios de mestizaje de la intertextualidad, la intermedialidad, y la autorreferencia auspiciados, entre otros fenómenos culturales, por un tipo de cine como es el de la adaptación de un videojuego. Los primeros se refieren a los solapamientos entre los diversos textos y entre los diversos lenguajes de ocasionados en la adaptación; el segundo concepto (autorreferencia) se enfrenta al fenómeno de la repetición y la 'serialidad' como rasgos predominantemente constitutivos en el régimen estético de la cultura de masas (Martínez, 12: 2013).

Cada adaptación se abrirá, en este sentido, tanto a múltiples direcciones y contextos, como a influencias pasadas y contemporáneas. El adaptador absorberá y reescribirá los géneros disponibles y los intertextos según el entorno (el discurso social dominante, el estilo del estudio que produce la película, las modas ideológicas, los condicionantes económicos, etc.). Son estas operaciones las que también se darán en el campo de la adaptación cinematográfica del videojuego.

Otro asunto es el margen de libertad creativa de la que dispone el adaptador para aplicar estas estrategias, y cómo estas se regulan según las indicaciones establecidas por los productores de la película. La 'adaptación' es hoy tanto un fenómeno intertextual e intermedial, como un modelo de negocio. La razones por las cuales la industria audiovisual, principalmente la de Hollywood, se han interesado por esta tipología de films van desde el factor económico e industrial de los grandes conglomerados mediáticos que controlan las productoras, al progresivo crecimiento de una audiencia interesada (o consumidora directa) de videojuegos, pasando por el avance de la tecnología y los efectos visuales que recogen ambas pantallas. 
Entre las variantes que ofrece el binomio cine y videojuegos (películas influenciadas por los videojuegos, películas alternativas o derivadas, etc.), la más sencilla de "detectar" es la adaptación de un videojuego al cine: cuando una productora cinematográfica descubre en una plataforma de juego las posibilidades de negocio/éxito en taquilla o en otras ventanas audiovisuales como el video casero/DVD o Internet.

En su tránsito hacia la gran pantalla, los videojuegos adaptados deben someterse a un proceso de traducción que les permita adecuarse a las operaciones y las estructuras habituales del cine. Por ejemplo, los juegos digitales deben prescindir de su potencial capacidad de desdoblamiento y de su prolongado tiempo de juego, para ceñirse a los límites de un largometraje cinematográfico y a las estructuras dramatúrgicas perennes en Hollywood. En este aspecto, deben adaptar su propio relato -muchas veces escaso (y, según casos, carente de profundidad)- así como las múltiples microestructuras narrativas que lo conforman entre niveles, para adecuarse al uso de la dramaturgia aristotélica (Riambau, 2011: 112) y a los estándares de este tipo de cine espectáculo producido por Hollywood: lo que Jullier (2004) denomina películas concierto, y Thompson (1981) cine del exceso o desmesura. Un cine "hiperabundante" de datos (Laferla, 2009), en el que la pantalla se llena de componentes estilísticos muchas veces superfluos para la unidad y el discurrir del relato (Darley, 2002). La Isla (Michael Bay, 2005), Speed Racer (Laurence y Andrew Wachowsky, 2008), Piratas del Caribe: En el Fin del Mundo (Gore Verbinski, 2007) o Avatar (James Cameron, 2009), son algunos de los ejemplos más representativos de la última década.

Asimismo, los videojuegos deben también lidiar con otra serie de mutaciones como las derivadas de su traducción estética (cómo la dirección artística de un film adaptará la del videojuego en la gran pantalla, cómo se trasladará la pulsión dinámica característica de la partida, etc.), o las derivadas de cuestiones económicas (no todos los presupuestos pueden reproducir con fidelidad el grado de espectáculo visual ofrecido por un videojuego). En definitiva, este desplazamiento hacia la pantalla cinematográfica supone para el juego tanto un conflicto interno, frente a su propia naturaleza y al mantenimiento de sus cualidades nativas, como externo, frente los múltiples factores económicos, mediáticos, estéticos, etc. que, además, mantienen otro pulso con el espectador. Como apunta Blanchet (2010):

Debemos situarnos en la intersección de dos industrias culturales que producen cada una contenidos de entretenimiento en masa [...] Una aproximación transdisciplinar que debe abrazar las diferentes dimensiones que 
cubren el cine y el videojuego, y que son, al mismo tiempo, dominios técnicos, industriales y económicos, objetos estéticos y prácticas sociales y culturales.

\section{Resultados}

\subsection{Dos categorías de adaptaciones cinematográficas de videojuegos}

Los modelos de adaptación cinematográfica de un videojuego pueden agruparse en dos grandes categorías definidas, principalmente, por el grueso de su inversión: las adaptaciones de alto presupuesto, que habitualmente adaptan juegos de gran éxito con los que la industria cinematográfica desea reproducir sus ventas en la gran pantalla. Y las adaptaciones de medio o bajo presupuesto, más discretas en su inversión y principalmente dirigidas a los aficionados del medio. A la primera pertenecen adaptaciones conocidas del entretenimiento digital como Super Mario Bros (Rocky Morton y Anabel Jankel, 1993), Street Fighter (Steven S. Souza, 1994), Tomb Lara Croft Raider (Simon West, 2001), Silent Hill (Christophe Gans, 2006), Max Payne (John Moore, 2008), Prince of Persia: Las Arenas del Tiempo (Mike Newell, 2010), etc. Todas ellas superan la media de los 30 millones de dólares en su producción. Mientras que la segunda agrupa juegos de menor "poderío" mediático en su propio ámbito y, por tanto, más encuadrados en el campo del aficionado a los videojuegos. Adaptaciones como Hitman (Xavier Gens, 2007), House of the Dead (Uwe Boll, 2003), BloodRayne (Uwe Boll, 2006), DOA: Dead or Alive. (Corey Yuen, 2006), etc. cuya producción, en general, se mantiene en un margen igual o inferior a los 25 millones de dólares.

En la conformación (y en algunos aspectos, interacción) de estas dos categorías generales, se puede destilar el interesado diálogo entre el mainstream cinematográfico y la segmentación de públicos que la industria de Hollywood practica para asegurar y ampliar el número de espectadores. Es decir, cuáles son las estrategias que esta industria pone en práctica para pasar de un producto en principio destinado a un público juvenil y conocedor de los videojuegos, a un producto blockbuster ${ }^{1}$ capaz de atraer a una gama más amplia de asistentes. 


\section{2 las adaptaciones de alto presupuesto}

En la primera categoría, las adaptaciones de alto presupuesto, se detecta un evidente empuje hacia la búsqueda de la rentabilidad internacional, apoyado en sumar al éxito mediático del videojuego factores como el reclamo de las estrellas de Hollywood o una producción apabullante que actúe también como señuelo. Este es un punto indicativo del por qué aproximadamente la mitad de las adaptaciones de videojuegos cuentan con actores conocidos (Bob Hoskins en Super Mario Bros, Angelina Jolie en Tomb Raider, Jean-Claude Van Damme en Street Fighter, Mila Jovovich en Resident Evil, etc.), directores solventes del cine espectáculo de Hollywood (Paul W. Anderson, Simon West) o productores de alto nivel como Jerry Bruckheimer (Prince of Persia: Las Arenas del Tiempo).

La práctica de la "alta inversión" en las adaptaciones de videojuegos ha ido asentándose progresivamente en el negocio cinematográfico desde su primero estreno, Super Mario Bros en 1993. La media de producciones cinematográficas que han pasado de los 30 millones dólares al adaptar un videojuego supera hoy la quincena. En conjunto, aquellas más exitosas en la taquilla han sido las películas pertenecientes a la saga Resident Evil y los films Prince of Persia: Las Arenas del Tiempo y Need for Speed. Todas ellas han sobrepasado tres veces (algunas hasta cuatro) su presupuesto inicial en el índice de recaudación mundial, rondando en esta recaudación un mínimo de 102 millones de dólares (Resident Evil) y un máximo de 336 millones (Prince of Persia: Las Arenas del Tiempo). Este dato, aunque no resulta significante si no se coteja con el presupuesto de los films, sí indica el considerable volumen de negocio y las ganancias que llegan a generar estas adaptaciones.

Las secuelas de la saga de Resident Evil y los films Prince of Persia: Las Arenas del Tiempo y Need for Speed pueden considerarse superproducciones de las adaptaciones de videojuegos ${ }^{1}$ Junto a ellas encontramos en este grupo de alta inversión una serie de adaptaciones con presupuestos estimados entre los 35 y 95 millones de dólares que, si bien no alcanzan el éxito en taquilla de las anteriores, sí alcanzan unas cotas de rentabilidad más que aceptables. Este espacio intermedio lo compone la secuela Tomb raider: La Cuna de la Vida (Jan de Bont, 2003) y las películas Silent Hill y Max Payne. La media de ganancias de estas producciones suele doblar su presupuesto inicial en la taquilla. Por último, en este grupo de superproducciones se define un final de lista compuesto por adaptaciones que, a pesar de contar con presupuestos de más de 50 millones de dólares, no consiguieron recuperar ni en el mejor de sus casos su inversión inicial. Se trata de sonados fracasos como los de Doom 
(Andrzej Bartkowiak, 2005), En el Nombre del Rey (Uwe Boll, 2007) o Street Fighter: The Legend of Chun-Li (Andrzej Bartkowiak, 2009).

Figura 1. Adaptaciones de alto presupuesto (periodo 1993-2014)

\begin{tabular}{|c|c|c|c|}
\hline $\begin{array}{l}\text { Adaptación } \\
\text { cinematográfica }\end{array}$ & $\begin{array}{l}\text { Presupuesto } \\
\text { Estimado } \\
\text { (dólares) }\end{array}$ & $\begin{array}{l}\text { Recaudación en } \\
\text { la cartelera } \\
\text { internacional } \\
\text { (dólares) }\end{array}$ & $\begin{array}{l}\text { Fecha } \\
\text { de Estreno }\end{array}$ \\
\hline Super Mario Bros & $48,000,000$ & $20,915,465$ & 1993 \\
\hline $\begin{array}{l}\text { Street Fighter: La } \\
\text { Última Batalla }\end{array}$ & $65,000,000$ & $12,077,284$ & 1994 \\
\hline Wing Commander & $30,000,000$ & $11,578,059$ & 1999 \\
\hline $\begin{array}{l}\text { Final Fantasy: La } \\
\text { Fuerza Interior }\end{array}$ & $137,000,000$ & $85,131,830$ & 2001 \\
\hline $\begin{array}{l}\text { Lara Croft: Tomb } \\
\text { Raider }\end{array}$ & $115,000,000$ & $274,703,340$ & 2001 \\
\hline Resident Evil & $33,000,000$ & $102,441,078$ & 2002 \\
\hline $\begin{array}{l}\text { Lara Croft: La Cuna } \\
\text { de la Vida }\end{array}$ & $95,000,000$ & $156,505,388$ & 2003 \\
\hline $\begin{array}{l}\text { Resident Evil: } \\
\text { Apocalipsis }\end{array}$ & $45,000,000$ & $129,394,835$ & 2004 \\
\hline Doom & $60,000,000$ & $55,987,321$ & 2005 \\
\hline $\begin{array}{l}\text { Resident } \\
\text { Extinción }\end{array}$ & $45,000,000$ & $147,717,833$ & 2007 \\
\hline En el Nombre del Rey & $70,000,000$ & $13,097,915$ & 2007 \\
\hline Max Payne & $35,000,000$ & $85,416,905$ & 2008 \\
\hline
\end{tabular}




\begin{tabular}{|l|l|l|l|}
\hline & & & \\
\hline $\begin{array}{l}\text { Street Fighter: La } \\
\text { Leyenda de Chun-Li }\end{array}$ & $50,000,000$ & $12,764,201$ & 2009 \\
\hline $\begin{array}{l}\text { Resident Evil: } \\
\text { Afterlife }\end{array}$ & $60,000,000$ & $296,221,663$ & 2010 \\
\hline $\begin{array}{l}\text { Prince of Persia: arenas del } \\
\text { Tiempo }\end{array}$ & $200,000,000$ & $336,000,000$ & 2010 \\
\hline $\begin{array}{l}\text { Resident Evil 5: } \\
\text { Venganza }\end{array}$ & $65,000,000$ & $240,159,255$ & 2012 \\
\hline \begin{tabular}{l} 
Need for Speed \\
\hline
\end{tabular} & $66,000,000$ & $203,277,636$ & 2014 \\
\hline
\end{tabular}

\subsection{Las adaptaciones de medio o bajo presupuesto}

En la segunda categoría, las adaptaciones de medio o bajo presupuesto, la directriz principal es rentabilizar a la audiencia específica interesada en el mundo de los juegos digitales, aprovechando juegos de éxito (aunque, en general, no tan reconocidos como los del grupo anterior), pero tomando muchos menos riesgos en cuanto a las inversiones de producción. Esta cuestión, por sí misma y en este campo cinematográfico, termina estableciendo una "serie B" en las mismas adaptaciones de videojuegos.

De tal manera, la envergadura del proyecto, así como sus estrategias de producción y difusión, dependerán tanto de la relevancia mediática y la rentabilidad del videojuego adaptado como del nivel establecido en el pre-sold project 2 por los productores e inversores del film. Todo ello no asegura la rentabilidad ni el éxito de la adaptación, pero permite establecer mejor las categorías de estudio donde analizar las consecuencias de estos éxitos o fracasos.

Según lo expuesto anteriormente, esta categoría de adaptaciones se sitúa en un margen de producción igual o inferior a los 25 millones de dólares. La conforman hoy una serie de films cuyas posiciones mediáticas y de rentabilidad económica son, en conjunto, menos potentes que el grupo 
anterior (una causa obvia debido a su baja financiación), y cuyos objetivos de público (el target potencial) y difusión funcionan, en principio y por estas mismas causas, con una distinta orientación. Se trata de proyectos de menor cuantía que, al tener menos posibilidades de ejecución, distribución y publicidad $^{3}$, planifican sus resultados financieros en base a la adquisición de los derechos de adaptación de un videojuego que les asegure, primeramente, un público base entendido en los videojuegos y, seguidamente, la posibilidad de amortizar este producto en el resto de ventanas de distribución (video/DVD y cadenas de TV).

De los films que componen este grupo, pocos han recuperado su inversión en la taquilla. Entre ellos, Hitman, que costó 24 millones de dólares ${ }^{4}$ y hoy su recaudación mundial se cifra en 99.965.792 dólares ${ }^{5}$; o Silent Hill 2: Revelación 3D (Michel J. Basset, 2012), que costó 20 millones de dólares y cuenta con una recaudación mundial actual de 52.302.796 dólares ${ }^{6}$. La tercera es House of the Dead que, frente a los 12 millones de dólares invertidos en su producción, salió del bache con una recaudación mundial de 13.818 .181 millones $^{7}$. El resto de la lista se compone de tropiezos económicos que ni siquiera lograron recuperar su propia inversión. Alone in the Dark (Uwe Boll, 2005) costó 20 millones de dólares y recaudó mundialmente 10.442 .808 dólares. DOA: Dead or Alive costó 21 millones de dólares y su recaudación se quedó en 7.516.532 dólares. BloodRayne (Uwe Boll, 2006) costó 25 millones de dólares y recaudó mundialmente 3.650.275 dólares. Y la controvertida Postal (2007), que se produjo con 11 millones de dólares, no llegó a alcanzar ni un solo millón: 146.741 dólares.

En lo que se refiere a la crítica cinematográfica, las muestras son igualmente decepcionantes. Resulta complicado encontrar un análisis en la prensa y en los medios especializados que defienda la ejecución o el discurso de alguna de estas adaptaciones. Lo habitual es encontrarse con un conjunto de diatribas, críticas o puntuaciones tan negativas como sus recaudaciones (sirvan al respecto, las puntuaciones obtenidas por estos films en webs especializadas como Film Affinty, IMDB o Rotten Tomatoes), basadas generalmente en la planicie de las propuestas y sus desenfrenos en el uso de los efectos especiales y las secuencias de acción.

Las concesiones, si es que aparecen, suelen recaer en algunos trasvases acertados entre videojuegos y cine, o incluso en las disparatadas decisiones que algunos cineastas adoptan para dan rienda suelta a los mismos excesos contenidos en la partida de juego (House of the Dead, Silent Hill 2: Revelación 3D). 
Cabe señalar también, y como en el caso de las adaptaciones de alto presupuesto, que muchas de estas producciones se etiquetan como runaways productions (coproducciones compartidas con el extranjero) y que comparten entre diversas productoras de Estados Unidos, Europa, Japón y Canadá sus gastos de financiación. Asimismo, que este grado medio o bajo de inversión ha permitido que, además de las participaciones de empresas consagradas del cine (20th Century Fox en el caso de la adaptación Hitman) o de los videojuegos (Infogrames en la adaptación de Alone in the Dark, Tecmo en el caso de la adaptación de $D O A$ : Dead or Alive), empresas incipientes de ambos medios hayan podido también hacerse un hueco en el área. Es el caso de la productora cinematográfica Mindfire Entertaiment con House of the Dead y su secuela, House of the Dead 2 (Michael Hurts, 2005) y DOA: Dead or Alive. O caso de la productora Herold Productions que ha financiado adaptaciones como En el Nombre del Rey, BloodRayne o Alone in the Dark.

Figura 2. Adaptaciones de medio o bajo presupuesto (periodo 19932014)

\begin{tabular}{|l|l|l|l|}
\hline $\begin{array}{l}\text { Adaptación } \\
\text { cinematográfica }\end{array}$ & $\begin{array}{l}\text { Presupuesto } \\
\text { Estimado } \\
\text { (dólares) }\end{array}$ & $\begin{array}{l}\text { Recaudación en } \\
\text { la cartelera } \\
\text { internacional } \\
\text { (dólares) }\end{array}$ & $\begin{array}{l}\text { Fecha } \\
\text { de Estreno }\end{array}$ \\
\hline Double Dragon & $7,850,000$ & $2,341,309$ & 1994 \\
\hline Mortal Kombat & $18,000,000$ & $122,195,920$ & 1995 \\
\hline $\begin{array}{l}\text { Mortal Kombat: } \\
\text { Aniquilación }\end{array}$ & $30,000,000$ & $51,376,861$ & 1997 \\
\hline House of the Dead & $12,000,000$ & $13,818,181$ & 2003 \\
\hline Alone in the Dark & $20,000,000$ & $10,442,808$ & 2005 \\
\hline DOA: Dead or Alive & $21,000,000$ & $7,516,532$ & 2006 \\
\hline BloodRayne & $25,000,000$ & $3,650,275$ & 2006 \\
\hline Hitman & $24,000,000$ & $99,965,792$ & 2007 \\
\hline Postal & $11,000,000$ & 146,741 & \\
\hline
\end{tabular}




\begin{tabular}{|l|l|l|l|}
\hline & & & \\
\hline $\begin{array}{l}\text { Silent Hill: Revelación } \\
3 D\end{array}$ & $20,000,000$ & $52,302,796$ & 2012 \\
\hline
\end{tabular}

\section{Discusión}

\section{1 Caso de estudio Lara Croft: Tomb raider}

Actualmente, la mayoría de las adaptaciones de alto presupuesto conforman un grupo compacto que, aún nivelado por los omnipresentes y repetitivos formulismos de Hollywood, ha sabido amoldarse regularmente a la taquilla y a las demandas del público. Su éxito sirve de modelo a las adaptaciones de medio o bajo presupuesto, las cuales, aun manteniendo el nivel económico y los rasgos propios de su categoría, adoptan generalmente estrategias cinematográficas similares para atraer al público. Es, por tanto, un producto de este grupo de mayor financiación (Lara Croft: Tomb Raider), el escogido en este artículo como caso de estudio que ejemplifica y concreta el estadio de las adaptaciones cinematográficas de videojuegos.

Los comienzos de tales adaptaciones, sin embargo, no fueron tan positivos. Se cuenta en un periodo inicial, entre 1993 y 2001, con sonados fracasos de crítica y taquilla, como Super Mario Bros, Street Fighter, Wing Commander (Chris Roberts, 1999) o Final Fantasy: La fuerza interior que, salvo excepciones (Mortal Kombat, Paul W.S. Anderson, 1995 y su secuela, Mortal Kombat: Aniquilación, John R. Leonetti, 1997), minaron la confianza de la industria cinematográfica y la producción de adaptaciones de juegos. Como muchas de las críticas especializadas indican ( pueden consultarse en fuentes citadas como Rotten Tomatoes o IMDB) al parecer, la causa principal de estos fracasos fue la prevalencia en estos films de la "forma" estética frente a cualquier otro aspecto a adaptar del juego. Es decir, adaptaciones que no atendieron verdaderamente al traslado de su narrativa, sus dinámicas o las experiencias del juego a la pantalla cinematográfica.

Es la superproducción Lara Croft: Tomb Raider, la que origina un giro de tendencias y reencamina este tipo de cine hacia un modelo de negocio rentable. Esto es, porque tras esta primera época de incertidumbre y ensayo, titilante entre fracasos de crítica y taquilla e inesperados aciertos o desaciertos (tanto entre films de media/baja inversión como los de mayor presupuesto), 
una gran apuesta de Hollywood sobre el videojuego consigue su propósito: generar beneficios siguiendo la estrategia establecida por la productora. Sin sorpresas y desde sus primeros estrenos (no como Street Fighter, cuya alta rentabilidad solo ha podido probarse casi una década después tras largo tiempo en distribución). Las cifras de esta adaptación del alto presupuesto pasan de los 115 millones de dólares que le costó el film a la Paramount, a los 274 millones resultantes en su recaudación internacional (sin contar otro tipo de beneficios derivados, por ejemplo, de la mercadotecnia $)^{10}$.

Todo ello sitúa a Lara Croft: Tomb Raider como el paradigma viable de la adaptación cinematográfica del videojuego a los ojos de la industria de Hollywood y su búsqueda del blockbuster efectivo y rentable. También, y tal y como se ha comentado, como un ejemplo y caso de estudio esencial para entender los funcionamientos de las adaptaciones cinematográficas de videojuegos.

Lara Croft: Tomb Raider recoge los aciertos de anteriores adaptaciones y los ejecuta con un éxito sin precedentes, convirtiéndose, a su vez, en un modelo posterior a imitar, tanto en lo que atañe a las adaptaciones de alto presupuesto, como en lo que se refiere a las adaptaciones de medio o bajo presupuesto. Esto se hace evidente en ulteriores adaptaciones de éxito de diferente presupuesto como, por ejemplo, las anteriormente citadas Resident Evil, Bloodrayne o Prince of Persia: Las Arenas del Tiempo. En ellas cuestiones como la escenografía, la iconografía sexual o el carácter "aventurero" (o de "acción”), se hacen patentes bajo el modelo establecido primeramente por Lara Croft: Tomb Raider .

La clave principal del film es la suma articulada del cine y el videojuego "de aventuras", junto a la confluencia "personificada" de dos icónicas figuras: la del personaje de videojuego Lara Croft, y la de la actriz destinada a encarnarla, Angelina Jolie. Lara Croft es un personaje diversamente atractivo tanto dentro como fuera del espacio del videojuego ${ }^{11}$.

Su reinterpretación visceral de la figura del aventurero (en especial del personaje de Indiana Jones creado por George Lucas y Steven Spielberg) en una forma más violenta y más sexual, era ya un reclamo de éxito en la saga de juegos y tenía todas las cualidades para triunfar en un film de sensaciones del tipo cine espectáculo. Más, con el añadido interpretativo de una actriz tan voluptuosa como Angelina Jolie; anzuelo morboso para los fans y los no entendidos del videojuego. Parte del atractivo de esta suma Croft-Jolie reside, sin embargo, en la masculinización temperamental a la que se somete su propio estereotipo femenino. Lara pasa de ampararse vigorosamente en símbolos fálicos (irguiendo su par de pistolas, derribando al robot Simon con un obelisco inmenso o metiéndose en orificios de esculturas), a mostrarse 
sensualmente desnuda mientras se ducha o mientras porta ceñidos atuendos que acentúan sus "hipersexualizadas" curvas. Además, corretea por el film, rodeada por un amplio grupo de actores "masculinos" (encabezado por el padre Lara ${ }^{12}$ y sus criados, y luego por sus aliados y villanos), que apenas deja espacio a otras figuras femeninas.

Otro aspecto que nos aproxima al éxito de Lara Croft: Tomb Raider es que la película, aunque algo prosaica, es una recreación muy próxima al juego que explota acertadamente la vertiente cinematográfica de éste mismo. Su cóctel de acción, mitología y sucesos paranormales (prácticamente calcado de las aventuras del citado Indiana Jones) se adapta sin problemas a un guión del cine espectáculo. Además, en su trasvase cinematográfico respeta el texto original y celebra los clichés estéticos que le dieron fama, en mayor medida que anteriores producciones de alto presupuesto como Super Mario Bros, o Street Fighter. De partida, este aspecto fílmico del juego es una gran ventaja: en comparación al resto de las anteriores adaptaciones, el film parte de un juego que ya poseía un notable espíritu cinematográfico inspirado en los films de aventuras del cine espectáculo tales como los del Indiana Jones o los del agente James Bond. Es decir, Lara Croft: Tomb Raider es una película que adapta un producto que ya de por sí se inspiró primeramente en la cinematografía de aventuras. Y estas películas de aventuras, a su vez, ya se habían fijado anteriormente en las novelas del género o en antiguos seriales (como en el caso de Indiana Jones, los producidos por Republic Pictures en la década de los 30).

Todo ello presenta un marcado caso de autorreferencialidad-de idea que pervive a lo largo del tiempo reformulándose en una diversidad de productos, que salta, en el caso de Lara Croft, primero del cine al videojuego, y luego del videojuego al cine. Esta idea recrea el concepto del aventurero/arqueólogo (sexy, en este caso, como los actores Harrison Ford y Angelina Jolie) que recorre múltiples escenarios salvajes y hostiles en busca de importantes tesoros. Una idea que ya había circulado previamente en otros muchos textos como por ejemplo, el videojuego Piffall! o las mismas versiones de los juegos de Indiana Jones de Lucas Arts, y que ha emergido recientemente en films como La Momia (Stephen Sommers, 1999), La búsqueda (Jon Turteltaub, 2004) o Las Aventuras de Tadeo Jones (Enrique Gato, 2012), entre muchos otros productos orientados a la "aventura arqueológica".

De este imaginario -o constructo cultural- y de la marcada intención de llevar el aspecto del videojuego al cine, surgen una multitud de referencias explícitas del tipo textual y formal (la intertextualidad e intermedialidad) que resaltan la misma interdisciplinariedad del producto. Si en el primer caso encontramos 
historias, escenarios y personajes muy semejantes a los que aparecen en la saga de juegos; en el segundo caso, cuando tratamos el tema formal, se pueden encontrar muchas adecuaciones del lenguaje del juego al cine y viceversa: una serie de ángulos de cámara que se desplazan como lo hacen en el juego (un deslizamiento espacial que acentúa la vertiginosidad de la acción y la majestuosidad de los entornos), continuadas acrobacias del personaje principal (nadar, saltar, escalar, disparar, etc.), decorados minuciosamente recreados como los del juego (como la mansión de Lara o los templos de Siberia y Camboya), etc.

El film Lara Croft: Tomb Raider también es responsable dentro del campo de las adaptaciones de videojuegos, del asentamiento de un modelo estándar ya practicado en la industria de Hollywood: La película preparada para la "estrella" cinematográfica. Anteriores superproducciones de las adaptaciones de videojuegos habían contado con estrellas de cine para interpretar a los personajes de la plataforma (Hoskins y Hooper en Super Mario Bros, Van Damme y Julia en Street Fighter, etc.), pero hasta la fecha ninguna había focalizado de tal manera su atención sobre el intérprete principal. La presencia absoluta de Angelina Jolie, como se ha comentado, funciona tanto para ensalzar la figura icónica de Lara Croft, como para explotar el reclamo del film. Visto el éxito de esta elección, Jolie repetirá posteriormente en la secuela Tomb Raider: La Cuna de la Vida (Jan de Vont, 2003), y marcará un precedente en próximas adaptaciones de videojuegos (tal y como hacen otras estrellas dentro del cine espectáculo). Si ella ocupó el metraje de Lara Croft, Mila Jovovich lo hará en la saga de films Resident Evil, Jake Gyllenhaal en Prince of Persia: Las Arenas del Tiempo, o Mark Walhberg en Max Payne. Todos son ellos son "estrellas" mediáticas atractivas para el público y, a su vez, capaces de asumir y permutar el rol icónico de un personaje de videojuego. Cabe señalar que las figuras de Jolie, Jovovich, Gyllenhaal, etc. han aportado y competido en la pantalla de cine tanto por el personaje que interpretan, como lo ha hecho su propia versión digital en el juego. El personaje digital de Lara Croft, fue remodelado en la siguiente entrega de la saga de juegos para asemejarse a Angelina Jolie, la figura de Mila Jovovich ha dado más fama (y más rostro) a la saga Resident Evil que muchos otros de los protagonistas virtuales de los juegos, etc ${ }^{13}$.

\section{Conclusiones}

Con todo, el que Lara Croft: Tomb Raider (y, a posteriori, su continuación Tomb Raider: La Cuna de la Vida) establezca un modelo económico relativamente rentable y, más adelante y a tales efectos, un modelo a seguir por otras adaptaciones de videojuegos, no significa que sea, desde el punto de vista de la 
crítica cinematográfica, un film acertado. Los resultados "artísticos" de los blocksbusters de Hollywood no suelen estar a la altura de sus presupuestos y Lara Croft: Tomb Raider, no resulta más que otro producto convencional del gremio. Quizás peor, ya que se trata de un producto que no cubre las expectativas o no está a la altura del original en lo que respecta a su equivalencia en la industria lúdica digital.

Resulta complicado comparar, las experiencias de un videojuego con las de una película; a todas luces, cada una presenta rutas particulares para el espectáculo y el entretenimiento. La dificultad (quizás imposibilidad) de trasvasar el nivel de intensidad y la sensorialidad del juego a la pantalla de cine es fácilmente discernible por quién consume estos dos productos. Quién ha jugado a la plataforma de juego ha experimentado la especial sinestesia y el dilatado tempo que se va construyendo al indagar por sus vastos y complejos escenarios, o el enervante -pero adictivo- pulso entre lo reflejos y habilidades "óculo-manuales" que nos exigen las escarpadas pruebas de cada nivel. Todas estas sensaciones y acciones son particularmente complicadas de traspasar a la butaca de cine: Por ello, al film Lara Croft: Tomb Raider siempre le faltará "cafeína" frente a la gameplay del videojuego.

Buena parte de los críticos y analistas profesionales de los videojuegos y del cine (Poole, Backzkowsky, Blanchet, etc.) apuntan a la complejidad -o imposibilidad- de estos trasvases. Pero no parece probable, sin embargo, que los realizadores del film Lara Croft: Tomb Raider y su secuela (o los de otras adaptaciones de videojuegos) sean ajenos a esta problemática. Sencillamente, y desde su apuesta por lo convencional y formulista del cine espectáculo, han intentado suplir estas faltas a través de otras facetas cinematográficas: la de la pirotecnia de la acción y los efectos especiales, y la del interés por la narración. Estos intentos, particularmente en Lara Croft: Tomb Raider y su continuación Tomb raider: La Cuna de la Vida, no pasarán a los anales de la historia, pero resultan solventes a efectos del gusto popular y la taquilla.

Resulta evidente, como también demuestran la mayoría de las adaptaciones de videojuegos, que está será siempre una losa sobre para los productos de este tipo: un empleo de la adrenalina y de la intensidad, distinto al que ofrece la experiencia de juego, más cuando el espectador ha degustado previamente la plataforma lúdica y puede compararla con su resultado cinematográfico. Pero ¿acaso no ha existido siempre una problemática equivalente en los traslados entre distintos formatos artísticos? El cine no puede comprender en su pantalla todas las viñetas, párrafos, improvisaciones $y$, en definitiva, experiencias, que plantean el cómic, la novela, el teatro y demás sujetos 
susceptibles de adaptación. Del latín adaptare, este término suscribe la misma esencia del trasvase: "ajustar a una cosa"; ni copiarla, ni trasladarla.

\section{Bibliografía y filmografía}

\subsection{Bibliografía}

BACKZKOWSKY, Melle Sandy (2005). La Contamination du Cinema Americain Contemporain par les Jeux Video. Universidad Toulouse-Le Mirail. Toulouse.

BLANCHET, Alexis (2010). Des Pixels a Hollywood. Cinéma et Jeu Vidéo. Une Histoire économique et Culturelle. Pixn' Love Editions.

BLANCHET, Alexis (2012). Les Jeux Vidéo au Cinéma. Armand Colin.

BORDWELL, David, THOMPSON, Kristin (1993) El Arte Cinematográfico. Paidós Comunicación. Barcelona.

BREA, José Luis (2003). "Estética, Historia del Arte, Estudios Visuales". Revista Estudios Visuales No 1. Cendeac.

$<$ http://estudiosvisuales.net/revista/index.htm> [Última consulta: 19/12/2015].

CASETTI, Franceso y DI CHIO, Federico (1990). Cómo Analizar un Film. Paidós. Barcelona.

DARLEY, Andrew (2002). Cultura Visual Digital. Espectáculo en los Medios de Comunicación. Paidós. Barcelona.

ECO, Umberto (1970). La Definición del Arte. Martínez-Roca. Barcelona.

GALLOWAY, Alexander (2007). "Acción del Juego, Cuatro Momentos". Jugabilidad: Arte, Videojuegos y Cultura. Artnodes No7. Universitat Oberta d Catalunya. < http://www.uoc.edu/artnodes/7/dt/esp/ $>$ [Última de consulta: 17/02/2011].

JULLIER, Laurent (2004). La Imagen Digital. De la Tecnología a la Estética. La Marca. Buenos Aires.

LA FERLA, Jorge (2009). Cine (y) Digital. Aproximaciones Posibles Convergencias entre el Cinematógrafo y la Computadora. Manantial. Buenos Aires. 
MARTÍNEZ, Mario-Paul (2013). Gameplay Abierta. Intertextualidad, Intermedialidad y Autorreferencia en la Partida de Juego. Revista Lifeplay: Investigación Y Videojuegos. Fundamentación y Bases Teóricas Del Videojuego. Universidad de Sevilla.

http://www.lifeplay.es/volumen1/revista.html. [Última de consulta: 30/06/2016].

McFARLANE, Brian. (1996). Novel to film: An Introduction to the Theory of Adaptation. Clarendon. Oxford.

MOXEY, Keith (2003). "Nostalgia de lo Real. La Problemática Relación de la Historia del Arte con los Estudios Visuales". Estudios Visuales No1.

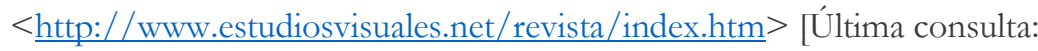
29/02/2015].

POOLE, Steven (2000). Trigger Happy: The Inner Life of Videogames. Arcade Publishing. Nueva York.

RIAMBAU, Esteve (2011) Hollywood en la Era Digital. De Jurassic Park a Avatar. Cátedra. Madrid.

STAM, Robert (2000). Teorías del Cine. Paidós Comunicación. Barcelona.

THOMPSON, Kristin (1981). Eisenstein's Ivan the Terrible: A Neoformalist Analysis. Princeton Univ Press. Princenton.

\subsection{Filmografía}

ANDERSON, Paul W. S. (2002). Resident Evil

ANDERSON, Paul W. S. (2010). Resident Evil: Afterlife.

ANDERSON, Paul W. S. (2012). Resident Evil: Retribution. Resident Evil 5: Venganza.

BARTKOWIAK, Andrzej (2005). Doom.

BARTKOWIAK, Andrzej (2009). Street Fighter: The Legend of Chun-Li. Street Fighter: La Leyenda de Chun-Li.

BASSET, Michael J. (2012). Silent Hill 2: Revelation 3D. Silent Hill 2: Revelación $3 D$. 
BOLL, Uwe (2003). House of the Dead.

BOLL Uwe (2005). Alone in the Dark.

BOLL, Uwe (2006). BloodRayne.

BOLL Uwe (2007). In The Name of the King: A Dungeon Siege Tale. En el Nombre del Rey.

BONT, Jan de (2003). Lara Croft: The Cradle of Life. Lara Croft: La Cuna de la Vida.

GANS, Christophe (2006). Silent Hill.

GENS, Xavier (2007). Hitman.

HURTS, Michael (2005). House of the Dead 2.

MORTON, Rocky y JANKEL, Annabel (1993). Super Mario Bros

MOORE, John (2008). Max Payne.

MULCAHY, Rusell (2007). Resident Evil: Extintion. Resident Evil: Extinción.

NEWELL, Mike (2010). Prince of Persia: The Sands of Time. Prince of Persia: Las Arenas del Tiempo.

ROBERTS, Chris (1999). Wing Commander.

SOMMERS, Stephen (1999). The Mummy. La Momia.

SOUZA, Steven S. (1994). Street Fighter. Street Fighter: La Última Batalla.

WEST, Simon (2001). Lara Croft: Tomb Raider.

\section{NOTAS}

${ }^{1} \mathrm{El}$ grueso de los Estudios del Juego referidos a las plataformas electrónicas y digitales nacen con el siglo XXI en el núcleo argumental de autores como 
Turkle, Poole, Galloway, Juul, Bogost, Aarseth, Zimmerman, Wolf, Perron, Jenkins, etc. quienes siempre han tenido en consideración la importante herencia de precursores como Callois o Huizinga, para edificar un campo hasta ahora austero y con lagunas. Sea por la pronta edad de estos estudios o por la retardada presencia de estas prácticas en los entornos académicos, los debates sobre la metodología de análisis de un videojuego no son numerosos (en comparación con los que atañen a otras artes) y para atender consecuentemente a su construcción, debe atenderse también a la aportación de varios autores clave, como los anteriormente citados, en este campo.

2 Producción cinematográfica de amplia inversión, encaminada a concebirse como una pieza asociada a un conjunto mayor de productos diversificados y, por consiguiente, a conseguir un mayor poder de convocatoria en el mercado y público (que, por ejemplo, una pieza de autor o que una película particular entendida dentro del régimen del Hollywood clásico).

${ }^{3}$ Cada una de ellas sobrepasa los 45 millones de dólares de presupuesto. Prince of Persia: Las Arenas del Tiempo llega incluso a barajar una producción estimada en millones de dólares.

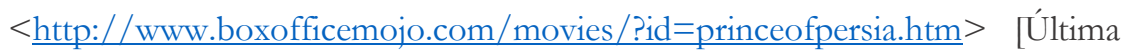
consulta: 15/06/2014].

${ }^{4}$ Como apunta Blanchet (2010: 71), un film en el que "la notoriedad se apoya sobre la popularidad de una obra preexistente asegurándose así la familiaridad con el espectador".

${ }^{5}$ No sólo hablamos de menor cuantía para producir un film, sino también de menor presupuesto para distribuirlo y, en especial, para publicitarlo. Las campañas mediáticas que apoyan a superproducciones como Prince of Persia o Lara Croft: Tomb Raider se encuentran a enormes distancias de films como House of the Dead, Hitman o BloodRayne, entre otros ejemplos de esta categoría de menor presupuesto.

$\begin{array}{llll}{ }^{6} \text { Estimación } & \text { realizada } & \text { por }\end{array}$ <http://www.imdb.com/title/tt0465494/?ref_=fn_al_tt_1> [Última consulta: 15/08/2014]. 
7<http://www.boxofficemojo.com/movies/?id=silenthill2.htm

đÚlima consulta: 15/08/2014].

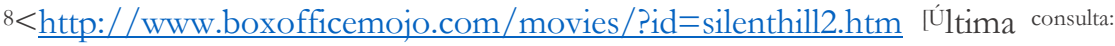
15/08/2014].

$9<$ http://www.boxofficemojo.com/movies/?id=houseofthedead.htm $>$ En la página del film de $I M D B$, en cambio, afirman que su presupuesto es de 7 millones de dólares. < http://www.imdb.com/title/tt0317676/> [Última consulta: 16/08/2014].

${ }^{10}$ Información extraída de Box Office: http://www.boxofficemojo.com/movies/?id=tombraider.htm Uúltima consulta: 04/04/2014]

${ }^{11}$ Es un icono del juego digital a la altura de figuras como Super Mario o PacMan. Ha sido adaptado al cómic (Top Cow Productions, 1999-2005), protagonista de varios anuncios publicitarios (anuncio del coche Seat Alambra en 2006), emulado por diversas modelos (en ferias de videojuegos como el E3) e incorporado en videoclips de bandas musicales de renombre como U2 (tema Elevation del disco All That You Can Leave Behind, 2000) o Korn (tema Did My Time del disco Take a Look in the Mirror, 2003).

12 Interpretado por su padre en la vida real, el actor Jon Voight.

${ }^{13}$ El papel icónico de Jolie no sufrió ningún cambio de Lara Croft: Tomb Raider a su secuela Tomb Raider: La Cuna de la Vida. Tampoco el esquema estipulado por el primer film. De nuevo, presenciamos un cóctel de acción, mitología y pirotecnia escenográfica. Y de nuevo, Lara reitera sus virtudes físicas y heroicas frente a las corporaciones enemigas, en la ya consabida persecución por un tesoro de consecuencias atroces para la humanidad. También encontramos una línea de producción semejante a la del primer film, con un director solvente en el área del cine de acción, Jan de Vont (responsable de clásicos del cine espectáculo como Speed, 1994 o Twister, 1996), al que le acompaña el guión de Dean Deorgaris y, esta vez sí en plantilla, Steven E. de Souza (director de Street Fighter y creador de una historia rechazada para el primer film de Tomb Raider) aparece en los créditos del guión/historia junto a James V. Hart. Como puede anotarse, las similitudes y referencias entre la primera y 
la segunda parte son tales, que prácticamente, pueden analizarse a la par. La mayor divergencia se refiere a la producción estimada, 95 millones de dólares, y al total de sus beneficios internacionales, 156.505.338 dólares; un resultado también positivo pero menor proporcionalmente al del primer film 


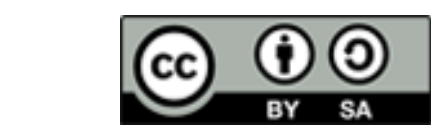

Licencia Creative Commons

Miguel Hernández Communication Journal

mhjournal.org

\section{Cómo citar este texto:}

Mario-Paul Martínez Fabre (2017): “Trasladar la partida: Modelos y estrategias de la industria cinematográfica frente a la adaptación de un videojuego", en Miguel Hernández Communication Journal, nº, pp. 205 a 229. Universidad Miguel Hernández, UMH (ElcheAlicante). Recuperado el $\_$de de 20_ de: @ink del artículo en mhjournal.org] 
